\title{
MODELING CHOICE STRATEGIES FOR NONCOMPARABLE ALTERNATIVES
}

\author{
MICHAEL D. JOHNSON \\ The University of Michigan
}

\begin{abstract}
Theoretical models are developed for two strategies consumers use to choose among "noncomparable" alternatives. The models view consumers as trading off decision error and processing effort when selecting a decision strategy. The models predict the use of choice strategies, and implicit abstraction processes, for noncomparable alternatives in a number of different circumstances.
\end{abstract}

(Comparability; Choice Strategies; Error/Effort Trade-Offs)

\section{Introduction: Comparability and Choice}

As consumer choice strategies affect both the quality of consumer decisions and the evaluation of particular products (Wright 1975; Russo 1981), understanding and anticipating such strategies may be central to the formulation of an effective marketing strategy. Our knowledge of strategy selection is generally limited, however, to descriptive studies of decisions involving very similar alternatives that are directly comparable. At the same time, marketers are becoming increasingly interested in issues related to across-category competition and product market boundaries (Day, Shocker, and Srivastava 1979; Srivastava, Alpert, and Shocker 1984).

This study focuses on an important and, until recently, unstudied set of consumer decisions involving more noncomparable or dissimilar consumption alternatives. In a recent study, M. Johnson (1984) recognized and investigated the problem of how consumers compare more noncomparable alternatives, such as a television and a stereo, or a television and a Hawaiian vacation. Comparability is defined as the degree to which consumers describe or represent alternatives using the same nonprice attributes, ranging from the very concrete to the very abstract (M. Johnson 1984, p. 741). The more alternatives overlap on descriptive attributes, the more inherently comparable they are. A stereo and a television are, for example, more noncomparable than either two stereos or two televisions; they are naturally described on many different attributes making direct comparisons more difficult.

M. Johnson proposed two general abstraction strategies by which consumers may compare these more noncomparable alternatives. First, independent of comparability, consumers may evaluate alternatives holistically, across their concrete attributes, in order to form and compare overall evaluations. Using a linear compensatory strategy (Einhorn 1970), for example, consumers may combine the screen size, picture quality, and price 
of a television and the length of stay, hotel location, and price of a Hawaiian vacation respectively into overall evaluations of "worth" or "utility" on which a comparison is made. The only comparisons that occur are among the highly abstracted overall evaluations; within-attribute comparisons are unnecessary.

Alternatively, consumers may redescribe or represent the alternatives on more general or abstract attributes in order to make direct, within-attribute comparisons. As more abstract attributes describe a greater number of alternatives, abstracting one's representation of alternatives to a level where comparability exists allows within-attribute comparisons. The more noncomparable the alternatives, the more abstract the required representations and resulting product comparisons. For example, a television and a stereo may be described and compared on "frequency of use" and "entertainment value," while a television and a Hawaiian vacation may require a description on "necessity" and "pleasure" in order to perform a within-attribute strategy such as additive difference (Tversky 1969) or elimination by aspects (Tversky 1972). M. Johnson (1984) reports the results of two empirical studies which support the use of these strategies. The subjects in the studies retained a within-attribute strategy by abstracting product representations to a comparable level of abstraction. They also made increasing use of across-attribute processing as comparability decreased.

Two important and related questions are addressed here. First, why do consumers use these strategies? Second, when might each strategy be expected? To help understand why consumers use these strategies, theoretically based models are developed. The models highlight the important differences and trade-offs between the basic abstraction processes involved, the absolute or holistic abstraction implicit in the across-attribute strategy and the representational abstraction required to use the within-attribute strategy. The models also predict the circumstances under which each process might be used. Such predictions may be particularly important to managers interested in product market boundaries or, put differently, the level of abstraction that separates particular products. For example, using the within-attribute strategy, even minor abstraction may suggest viable consumption alternatives in the minds of consumers. In the next section of the paper, a general framework for modeling these processes is described.

A related yet separate issue concerns what Slovic and MacPhillamy (1974) refer to as dimensional commensurability. Slovic and MacPhillamy found that decision makers place more weight on the commensurable dimensions of the choice (those on which all alternatives have values) relative to dimensions on which attribute values could exist but are missing for a subset of alternatives. In a similar study, Yates, Jagacinski, and Faber (1978) found that the existence of missing values lowers the evaluation of choice alternatives. They suggest that the uncertainty associated with such alternatives results in devaluation. Commensurability is, nonetheless, qualitatively different from comparability. Both Slovic and MacPhillamy and Yates et al. assume a common, or comparable, set of attributes. The question here is how more abstract representations or evaluations are formed for alternatives varying in comparability given the existence of complete, concrete product representations.

\section{A Positive Approach to Strategy Selection}

To formulate a more positive, theoretical approach to strategy selection, one must begin by considering consumers' goals when selecting a strategy. Consistent with previous studies, assume that consumers wish to minimize both decision error and processing effort when selecting a decision strategy (Hogarth 1975; M. Johnson 1980; Russo 1981; Shugan 1980; Russo and Dosher 1983). Because, however, minimization of error and effort are incompatible goals, consumers must trade-off error for effort. A decrease in error is generally obtained only through an increase in decision making effort. Likewise, a decrease in effort is possible only by accepting a greater possibility of error. 
This approach is conceptually consistent with other models of strategy selection based on a contingency or cost-benefit analysis between the decision maker's desire to make a correct decision and the time and effort involved (Beach and Mitchell 1978). As in the error-effort research, the time, energy, and other related psychological and monetary costs associated with different decision strategies are considered costs to the decision maker. Each strategy is, in turn, associated with some expected benefit, or level of decision making error. Optimal strategies are those that offer the greatest expected net gain. This general cost-benefit model has been formalized and tested in a number of experimental situations (Christensen-Szalanski 1978, 1980; McAllister, Mitchell, and Beach 1979; Smith, Mitchell and Beach 1982) and has been shown to predict many aspects of strategy selection. Christensen-Szalanski (1978), for example, showed how an increase in the benefit of making a correct decision results in more time (and presubably effort) being allocated to the decision. In a more recent study, Christensen-Szalanski (1980) found that differences in analytic ability (business versus nonmath student subjects) result in very different decision making costs.

This is not to say that this error-effort or cost-benefit trade-off holds in every situation. For example, minimizing effort by choosing randomly may be one way of efficiently gathering information in an unfamiliar environment in order to make good decisions, or minimize error, in the future (March 1978). Therefore, while the error-effort tradeoff is assumed here, naturally there will be situations where strategy selection does not follow a cost-benefit trade-off (Einhorn and Hogarth 1981) or where minimization of error and effort are not incompatible (March 1978). It is also dangerous to assume that such a trade-off is explicitly calculated by consumers. As E. Johnson and Payne (1985) note, if we assume that people deliberately decide how to choose, there is a potential infinite regress, where "One has to decide how to choose to decide how to choose . . ." (p. 29). Nevertheless, decision making research to date suggests that the cost of thinking is important to consider when modeling strategy selection. It seems more realistic, however, to consider the error-effort trade-off as either a rough calculation made at the time of the decision (Russo 1981) or, though not explicitly determined, learned over time (Klein 1983).

\section{Strategy Models for Noncomparable Alternatives}

The present study goes beyond existing strategy selection research by explicitly modeling the basic abstraction processes or strategies consumers use to compare more noncomparable alternatives. The key concepts and trade-offs between the two forms of abstraction are made explicit. Once explicit, operationalization of the models provides predictions regarding strategy selection across situations which vary with respect to the comparability of the choice alternatives and other consumer- and task-related variables, including consumer knowledge, the number of alternatives, and the number of choice-relevant attributes at different levels of abstraction.

\section{Strategies Modeled and General Processing Assumptions}

As the focus here is on abstraction processes for noncomparable alternatives, only the two strategies studied by M. Johnson (1984) are modeled. The particular within-attribute strategy modeled is equivalent to a compensatory additive difference strategy ${ }^{1}$ (Tversky 1969 ) with an added abstraction stage of processing. There are three stages required for this strategy. First, the alternatives must be represented on nonprice attributes at a comparable level of abstraction. Direct comparisons are then made on both the nonprice attributes at that level and price. Finally, the resulting relative attribute comparisons

\footnotetext{
' The additive difference strategy need not be strictly compensatory. Both Tversky (1969) and Russo and Dosher (1983), for example, describe heuristic versions of the model that are only globally compensatory.
} 
must be combined into a relative overall comparison on which a choice is made. The across-attribute strategy modeled is equivalent to a compensatory additive utility strategy (Einhorn 1970), where all concrete nonprice attributes are combined along with price into overall evaluations. The overall evaluations are then directly compared to make a choice.

Less effortful elimination and phased strategies are not considered. Strategies involving only compensatory processing were chosen for three reasons. First, as the goal here is to focus specifically on abstraction processes for noncomparable alternatives, it is assumed that consumers process the same amount of relevant decision information in the same compensatory manner in order to hold these factors constant. Second, studies looking at the details of choice strategies, or process tracing studies, have found processing to be generally compensatory when the number of alternatives involved in a choice is relatively small (cf. Lussier and Olshavsky 1979; Payne 1976; Russo and Dosher 1983; Russo and Rosen 1975). (As the number of alternatives grows, processing is less likely to be compensatory. Therefore, while the number of alternatives involved in a choice is modeled, only relatively small values are considered here $(n \leq 6)$.) Finally, the difference between compensatory and noncompensatory strategies, or strategies which process varying amounts of information, is already the focus of a body of decision making research (cf. Beach and Mitchell 1978; M. Johnson 1980; Thorngate 1980; E. Johnson and Payne 1985).

For both strategies, a mechanism by which consumers reduce more than two alternatives to a single alternative must be specified. An efficient elimination mechanism which decision makers have been found to use, or at least approximate, in a number of studies is termed standard revision (Russo and Rosen 1975; Payne 1976; Lussier and Olshavsky 1979). According to standard revision, multiple alternatives are compared in a pairwise fashion. One alternative is eliminated with each binary comparison resulting in $(n-1)$ binary comparisons for $n$ alternatives in order to make a choice. Given both its theoretical appeal and empirical support, standard revision is assumed for each strategy.

To keep the models tractable, other assumptions are made. First, consumers are assumed to have equal knowledge of the choice alternatives. Knowledge does, however, vary across individuals and choices. Second, all nonprice attributes are represented at a single level, $i$, of abstraction. Third, alternatives are assumed to have an equal number of nonprice attributes at a given level of abstraction, although the number of attributes may differ across levels and from choice to choice. The sole purpose of these assumptions is simplicity. They allow the choice alternatives to be modeled as a group at a single level of abstraction. The assumptions may be relaxed by modeling the alternatives individually and at multiple levels. Finally, price is assumed not to vary in level of abstraction. Price is a concrete attribute characterizing most consumption alternatives. Even alternatives that are noncomparable on nonprice attributes are perfectly comparable on price.

\section{Effort}

Given the interest in choice strategies, effort is confined to the cognitive resources required to complete the choice task (Russo and Dosher 1983). Following the suggestion of Newell and Simon (1972), previous studies have attempted to decompose these cognitive resources down into a particular unit or units of thought (Huber 1980; E. Johnson and Payne 1985; M. Johnson 1980; Shugan 1980). Strategies can then be thought of as requiring a series of such units, or elementary information processes (EIP's; see Chase 1978). One benefit of this approach is that the effort required to perform particular strategies can be operationalized by looking at the total number of EIP's required by each strategy (Newell and Simon 1972). M. Johnson (1980), for example, used this procedure to look at the error and effort involved in several choice heuristics for analyzing product label information. Both Huber (1980) and E. Johnson and Payne (1985) use a 
similar technique, Huber in the context of choice between job candidates and Johnson and Payne in the context of gambles.

The procedure used here builds on this approach by including both previously used EIP's (e.g. the number of attribute values compared or recalled) and additional necessary operations that are unique to the strategies modeled here (e.g. the number of levels abstracted to form a comparable representation). In an attempt to remain parsimonious, only the EIP's and other aspects of abstraction on which the decision strategies differ are modeled.

\section{The Error/Effort Trade-Off}

In the process of selecting a decision strategy, both decision error and processing effort can be conceptualized as sources of disutility or costs that consumers wish to minimize. The constraint placed on this objective is that a decrease in the disutility of one factor is accomplished only by allowing the disutility of the other to increase. That is, by expending effort and processing relevant information, consumers reduce the expectation of error. One can, therefore, view the consumers goal as one of minimizing overall disutility by selecting a decision strategy, where the overall expected disutility $(E D)$ of any particular strategy is,

$$
E D=E D(E R)+E D\left(E F_{i}\right) \quad \text { and }
$$

$i=$ level of abstraction of information,

$E D(E R)=$ the expected disutility making an error,

$E D\left(E F_{i}\right)=$ the expected disutility of effort expended processing information at $i$.

This overall disutility is minimized subject to the following constraint:

$$
E D(E R)=\left[U-U_{\text {max }}\right]\left(1-E F_{i} / E F_{i, \text { ideal }}\right) \quad \text { where }
$$

$U_{\max }=$ utility of the best alternative,

$U=$ average utility of all alternatives in the choice set,

$E F_{i}=$ effort expended processing information at $i$,

$E F_{i \text {, ideal }}=$ effort required to make an ideal choice at $i$.

Equation (2) takes as a baseline or theoretical limit that consumers choose at random when no effort is expended on a choice, or $E F_{i}$ equals zero. The expected overall disutility that results is simply the difference between the utility gained by choosing an average (random) alternative and the utility gained by choosing the best possible alternative, or $\left[U-U_{\max }\right]$. As effort increases and approaches an ideal level, $E F_{i, \text { ideal }}$, the expected disutility of error approaches zero.

While straightforward, this measure of error assumes that incremental effort has equal error reducing value. While the relationship between error and effort is problematic and, in fact, may often be nonlinear, ${ }^{2}$ for the purposes of this study (which does not focus specifically on the effects of incremental effort), this simple relationship is maintained.

As expending effort adds to the cost of deciding, substituting equation (2) into equation (1) yields the following overall error and effort disutility of deciding using any particular strategy:

$$
E D=\left[U-U_{\max }\right]\left(1-E F_{i} / E F_{i, \text { ideal }}\right)+E D\left(E F_{i}\right) .
$$

\section{Within-Attribute Strategy}

In order to specifically model the within-attribute strategy, we must identify those components on which this strategy differs from the across-attribute strategy. These include

\footnotetext{
${ }^{2}$ As certain strategies, such as the lexicographic rule (Coombs 1964), process more relevant or important attributes first, incremental effort under such strategies results in decreasing incremental reductions in error. As attribute information is also often very redundant or highly correlated, even processing equally important attributes often results in decreasing returns from effort (Einhorn, Kleinmuntz, and Kleinmuntz 1979).
} 
representational abstraction, comparison, and combination effort. Each of these components is modeled independently.

\section{Representational Abstraction Effort}

Following M. Johnson (1984), representational abstraction in the within-attribute strategy is considered a two-stage process. Consumers must first construct a set of applicable attributes at the desired level of abstraction (one at which the alternatives are comparable). Consumers may then form values for the alternatives on the overlapping attributes. Choosing between a television and a vacation, for example, one first sees the alternatives differing on "necessity" and "practicality" and then tries to determine the particular values for the alternatives on these dimensions. These more abstract, common attributes may be formed by moving to the common superordinate or categorical node in memory for the alternatives (Quillian 1969; Collins and Loftus 1975). The justification for such a process is purely theoretical. While there are possible alternatives to the twostage model (for a discussion see M. Johnson 1985), differences focus more on the possible order of processing rather than on the operations that are required to form an abstract representation.

Naturally, abstraction effort should depend on a consumer's knowledge of product attribute values. Knowledgeable consumers are more able to simply recall abstract attribute values, such as the necessity or practicality of a television, from memory. More naive consumers, in contrast, must construct such values. Attribute value construction implies some sort of "mapping" of the available concrete attribute values into values on the more abstract attributes.

As no research is available to indicate otherwise, the simplest and most justifiable assumption is that each attribute constructed, attribute value recalled, and attribute value mapped has an independent effect on effort (Newell and Simon 1972). Effort is assumed to increase linearly with the number of attributes, attribute values, and alternatives involved. Effort disutility to form a more abstract representation at level $i, E D\left(E F_{A i}\right)$, can then be measured by

$$
E D\left(E F_{A i}\right)=b_{1} n m_{i}+\theta_{i}\left(b_{2} n m_{i}\right)+\left(1-\theta_{i}\right)\left(b_{3} n m_{0} a_{i}\right) \quad \text { where }
$$

$n=$ number of alternatives in the choice,

$b_{1}=$ disutility of constructing one attribute for one alternative,

$m_{i}=$ number of relevant nonprice attributes per alternative at level $i$,

$\theta_{i}=$ degree to which attribute values are recallable, from 0 to 1 ,

$b_{2}=$ disutility of recalling one attribute value for one alternative,

$b_{3}=$ disutility of mapping one attribute value of one alternative over one level of abstraction,

$a_{i}=$ number of levels abstracted from 0 to $i$.

Equation (4) contains three component parts, attribute construction, value recall and value construction respectively. In the first component, effort increases linearly with the number of attributes and alternatives in the representation. In the second component, effort increases linearly with the number of attribute values recalled. In the third, effort increases linearly with both the number of concrete values that must be mapped into more abstract attributes and the number of levels of abstraction over which the mapping occurs. $^{3}$

\footnotetext{
${ }^{3}$ While the first two components, attribute construction and value recall, are straightforward, value mapping is more problematic. Two assumptions are implicit in equation (4) regarding this process. First, effort to map values increases linearly with the number of levels of abstraction over which the mapping occurs. While there is no research which bears on the validity of this assumption, it appears logical. Consider, as an example, that estimating the "pleasure" of a television from its "screen size" is more effortful than estimating its "entertainment value" from "screen size" which, in turn, is more effortful than estimating "picture quality" from "screen size."
} 
As knowledge increases, ability to recall values increases relative to one's need to construct or map such values. Therefore, effort to form values on the attributes in the representation is modeled in equation (4) as a weighted average of recalling versus mapping. The weight, $\theta_{i}$, represents relative ability to recall, which varies from 0 (no recall) to 1 (complete recall) as knowledge increases. Note that $b_{1}, b_{2}$, and $b_{3}=0$ when $i=0$, and $b_{1}, b_{2}$, and $b_{3}>0$ when $i>0$ (i.e., when the consumer stays at a concrete level, there is no representational abstraction effort). Also, the model assumes that both the recall and construction of values is accurate (i.e. there is no error as a result of recalling or mapping).

In sum, abstraction effort has three components. First, a set of attributes must be constructed that apply to each of the alternatives at the desired level of abstraction. Second, values on attributes for the alternatives are recalled from memory. Third, values that cannot be recalled are constructed by mapping values on concrete attributes into values on the abstract attributes.

\section{Evaluation Effort}

Evaluation effort includes all attribute comparisons and combinations necessary to perform an additive difference evaluation on the now comparable alternatives. Recall that, by standard revision, $(n-1)$ pairs of alternatives are compared. For each pair, $m_{p i}$ attribute comparisons occur per pair, where $m_{p i}$ is the total number of price and nonprice attributes processed at $i$. Of course, $m_{p i}$ equals $\left(m_{i}+1\right)$ when price and all relevant nonprice attributes are processed. These relative comparisons are then combined to allow a choice to be made between the two alternatives. Reference to a combination process does not imply that consumers literally combine values. The combinations inherent in additive difference, linear compensatory, and other choice models are consistent with the psychological process of anchoring and adjusting (Tversky and Kahneman 1974; Lopes and M. Johnson 1982; see also Einhorn and Hogarth 1981). Such a process results in $\left(m_{p i}-1\right)$ combinations or "adjustments" per choice pair. If, for example, $m_{i}$ equals three, comparisons occur on three nonprice attributes and on price. Consumers anchor on one of these four differences and then adjust their judgment of relative worth by subsequently considering the second, third, and fourth comparisons. As comparisons and combinations occur for each pair of alternatives involved, $(n-1) m_{p i}$ attribute comparisons and $(n-1)\left(m_{p i}-1\right)$ combinations occur overall. Again, a simple linear combination of comparisons and combinations provides a measure of evaluation effort at level $i$.

Therefore, combining disutility of error, abstraction effort, and evaluation effort into an overall weighted disutility of using the within-attribute strategy at $i, E D\left(W_{i}\right)$, we obtain:

$$
\begin{aligned}
& E D\left(W_{i}\right)=\left[U-U_{\max }\right] {\left[1-E F_{i} / E F_{i, \text { ideal }}\right]+n\left[b_{1} m_{i}+\theta_{i} b_{2} m_{i}\right.} \\
&\left.+\left(1-\theta_{i}\right) b_{3} m_{0} a_{i}\right]+(n-1)\left[b_{4} m_{p i}+b_{5}\left(m_{p i}-1\right)\right] \quad \text { where }
\end{aligned}
$$

$b_{4}=$ disutility of making a relative comparison,

$b_{5}=$ disutility of combining two relative evaluations, and

$m_{p i}=$ number of attributes processed at $i$.

The parameters used to model both the within-attribute strategy with abstraction and the across-attribute strategy are listed for the reader in Table 1. 
TABLE 1

Summary of Parameter Descriptions, Modeling Assumptions, and Operationalized Values

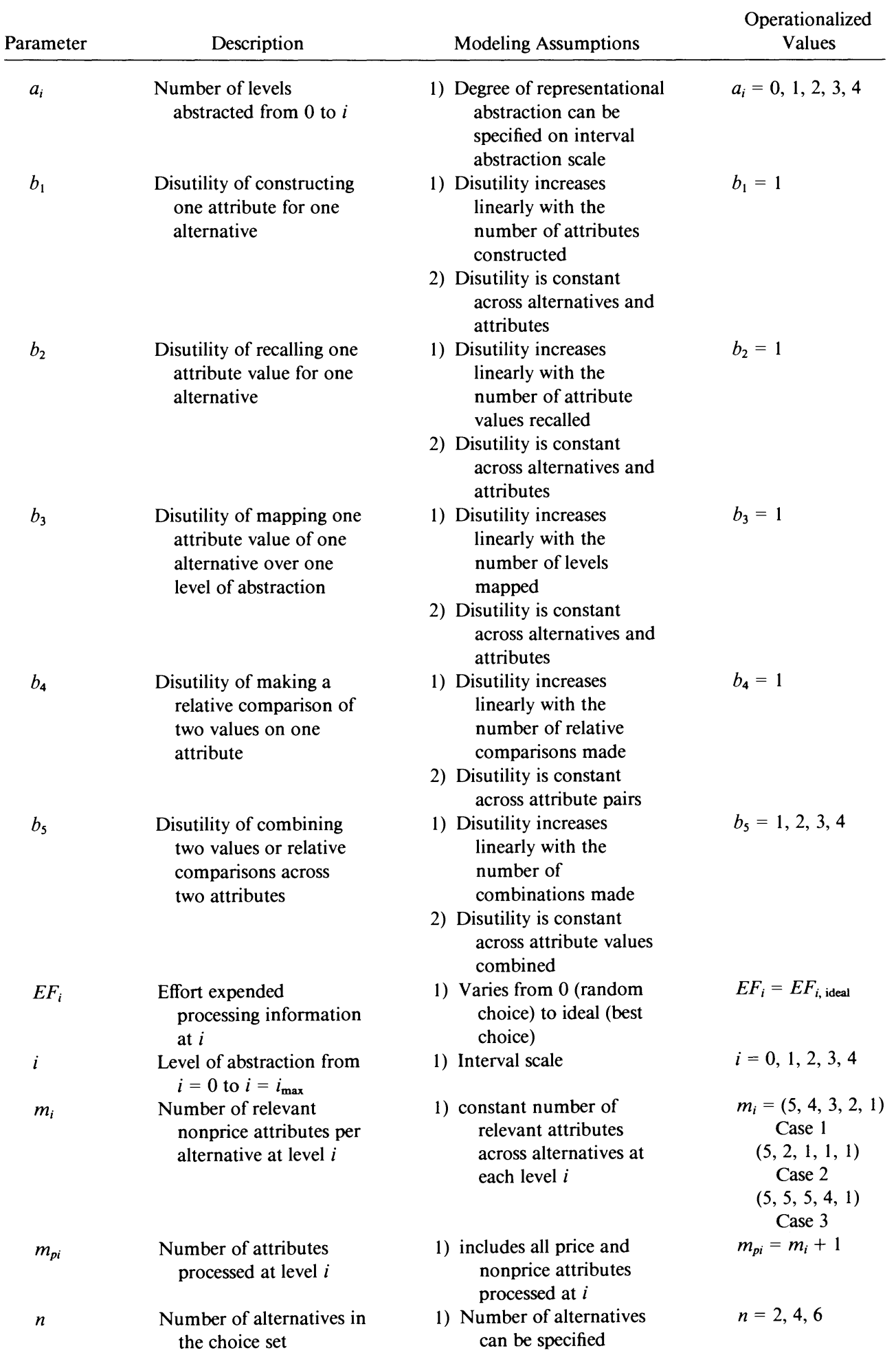


TABLE 1 (Continued)

\begin{tabular}{|c|c|c|c|}
\hline Parameter & Description & Modeling Assumptions & $\begin{array}{c}\text { Operationalized } \\
\text { Values }\end{array}$ \\
\hline$\theta_{i}$ & $\begin{array}{l}\text { Degree to which attribute } \\
\text { values are recallable } \\
\text { from } 0 \text { to } 1\end{array}$ & $\begin{array}{l}\text { 1) Ability to recall abstract } \\
\text { attribute values } \\
\text { increases } \\
\text { monotonically with } \\
\text { consumer knowledge }\end{array}$ & $\theta_{i}=0,0.5,1$ \\
\hline$U$ & $\begin{array}{l}\text { Average utility of } \\
\text { available choice } \\
\text { alternatives }\end{array}$ & $\begin{array}{l}\text { 1) Utility received when } \\
\text { choice is random (no } \\
\text { processing effort is } \\
\text { expended) }\end{array}$ & No Estimate \\
\hline$U_{\max }$ & $\begin{array}{l}\text { Utility of maximum } \\
\text { utility alternative in } \\
\text { the choice set }\end{array}$ & $\begin{array}{l}\text { 1) Utility received when best } \\
\text { alternative is chosen } \\
\text { (all relevant } \\
\text { information is } \\
\text { processed) }\end{array}$ & No Estimate \\
\hline
\end{tabular}

\section{Across-Attribute Strategy}

The across-attribute strategy assumes that processing occurs on concrete attributes at $i=0$. This strategy does not require representational abstraction. The only abstraction process here is that involved in the combination of attribute values into overall evaluations.

Two important observations are made concerning this evaluation effort. First, a combination is similar to the two strategies. In the across-attribute strategy, combinations are across absolute attribute values (e.g., a 25 -inch television screen "plus" a clear picture) as opposed to relative attribute values for the within-attribute strategy (e.g., a larger television screen "plus" a clearer picture). Using different inputs may cause a difference in disutility per combination in the two strategies. In both cases, however, the process of anchoring and adjustment is likely to be used (Einhorn and Hogarth 1981). The task is the same ("combining apples and oranges") and any differences are likely to be small. Second, a comparison is identical in the two strategies. In the across-attribute strategy, one comparison is made on overall evaluations for each pair of alternatives as opposed to one or more comparisons on descriptive attributes in the within-attribute strategy. Assuming that the disutility of comparing and combining is identical across strategies, $b_{4}$ and $b_{5}$ are taken directly from the within-attribute strategy. While detailed research might reveal differences in these values across strategies, for the sake of parsimony, we reserve this issue for further research. Again, disutility is assumed linear with respect to combinations, comparisons, and alternatives processed.

The strategies differ in terms of the number of combinations and comparisons involved. Using the across-attribute strategy, each of the $n$ alternatives has each of $m_{p 0}$ attributes combined to form overall evaluations resulting in $n\left(m_{p 0}-1\right)$ total combinations. Notice that this implies more combinations than the within-attribute strategy when alternatives are comparable. This is consistent with Tversky's (1969) justification regarding the relative ease of within-attribute processing. Once combined, the $n$ overall evaluations are compared resulting in $(n-1)$ comparisons, assuming a standard revision process. Combining combination and comparison disutility with the error-effort trade-off at $i=0$ yields the overall disutility to perform the across-attribute strategy at level $0, E D\left(A_{0}\right)$ :

$$
E D\left(A_{0}\right)=\left[U-U_{\text {max }}\right]\left[1-E F_{0} / E F_{0, \text { ideal }}\right]+\left[b_{4}(n-1)+b_{5} n\left(m_{p 0}-1\right)\right] .
$$




\section{Strategy Differences and Trade-Offs}

Considering the difference in disutility between strategies reveals the trade-offs involved in the strategy selection process. The disutility difference between strategies, $E D\left(W_{i}\right)$ $-E D\left(A_{0}\right)$, becomes

$$
\begin{array}{r}
E D\left(W_{i}\right)-E D\left(A_{0}\right)=\left[U-U_{\text {max }}\right]\left[E F_{i} / E F_{i, \text { ideal }}-E F_{0} / E F_{0, \text { ideal }}\right]+n\left[b_{1} m_{i}+\theta_{i} b_{2} m_{i}\right. \\
\left.+\left(1-\theta_{i}\right) b_{3} m_{0} a_{i}\right]+b_{4}(n-1)\left(m_{p i}-1\right)+b_{5}\left[(n-1)\left(m_{p i}-1\right)-n\left(m_{p 0}-1\right)\right],
\end{array}
$$

where $b_{1}, b_{2}, b_{3}=0$ when $i=0 ; b_{1}, b_{2}, b_{3}>0$ when $i>0$; and $b_{4}, b_{5}>0$ for $i \geq 0$. This difference predicts optimal disutility minimizing strategies across contexts. When the difference exceeds zero, the across-attribute strategy minimizes disutility; when equal to zero, disutility is equal; when less than zero, the within-attribute strategy minimizes disutility. Whichever strategy minimizes overall disutility is selected.

\section{Operationalizing the Models}

By operationalizing the models and equation (7), the abstraction processes that minimize overall disutility in particular contexts can be determined and strategy selection can be predicted. Two qualitatively different types of parameters must be operationalized, those that describe the task situation and those that describe the consumer.

\section{Task Parameters}

The task facing the consumer is described by (1) the comparability of the alternatives, (2) the number of alternatives involved, and (3) the number of relevant attributes at different levels of abstraction. Previous studies of the effects of task complexity have systematically varied the number of alternatives and attributes involved in a choice (Payne 1976; Lussier and Olshavsky 1979). The comparability of alternatives is added here, and various levels of these parameters are used to identify various contexts in which strategy selection may occur.

Comparability is operationalized by (1) operationalizing the within-attribute strategy model at different levels of $a_{i}$ (the number of levels abstracted from 0 to $i$ ) and (2) assuming that the within-attribute strategy is performed at a level of abstraction equal to or greater than that at which comparability exists. For example, if choice alternatives are moderately noncomparable, then use of the within-attribute strategy at only intermediate levels of abstraction and above (those levels at which the alternatives are comparable) is considered.

Obviously, in order to operationalize comparability, the number of levels of abstraction possible, from $i=0$ to $i=i_{\max }$, must be specified. Five levels of abstraction are used here, $a_{i}=0,1,2,3$, and 4 . As a rationale, consider that as representations become more abstract, the number of choice-relevant attributes should decrease. The empirical results of both Boote (1975) and M. Johnson (1984) support this contention. At the same time, consumers seldom use more than four or five attributes when evaluating alternatives (Jacoby, Szybillo and Busato-Schach 1977; Lussier and Olshavsky 1979; Brucks 1985). If, for example, relevant attributes decrease by one from level to level, then at least four or five levels of $i$ are necessary. At the same time, using more than five levels seems unnecessary. As, however, the use of five levels is relatively arbitrary, the models are also operationalized assuming both three and ten levels respectively to determine the sensitivity of the results to this parameter.

The number of alternatives is examined at three levels, $n=2,4$, and 6 . These values are kept small as, again, the compensatory strategies modeled in equation (7) are more likely when $n$ is relatively small. 
The number of relevant attributes at different levels of abstraction is described by three possible cases. Case 1 represents an "average" case, a linear decrease from level to level where $\left(m_{0}, m_{1}, m_{2}, m_{3}, m_{4}\right)=(5,4,3,2,1)$. Two other cases represent extremes in the relationship between $m_{i}$ and $i$ which may better approximate consumer choices. Consumer choice among nondurable goods generally involves little risk and, it seems, few relevant attributes above a very concrete level of abstraction. Food products, for example, may be evaluated on several concrete attributes (e.g. sweetness, fat content, sodium level) though very few even moderately abstract attributes are relevant (e.g. nutritional value). Durables, in contrast, represent alternatives for which several even moderately abstract attributes may be relevant. Deciding between a television and a refrigerator, for example, may involve important differences on moderately abstract attributes such as frequency of use, social status, entertainment value, and attractiveness. Thus the number of relevant attributes should decrease more rapidly from the concrete to the abstract for nondurable products than for durable products. Resembling nondurables, Case 2 represents a very drastic decrease in relevant attributes where $\left(m_{0}, m_{1}, m_{2}, m_{3}, m_{4}\right)=(5,2,1,1,1)$. Resembling durables, Case 3 represents a very gradual decrease where $\left(m_{0}, m_{1}, m_{2}, m_{3}\right.$, $\left.m_{4}\right)=(5,5,5,4,1)$.

\section{The Consumer}

The error and effort parameters of the consumer include the level of effort put forth processing information, the ability of the consumer to recall abstract attribute values, and the values of the various disutility parameters in equation (7). As stated earlier, the goal here is to focus specifically on the differences between abstraction processes for evaluating noncomparable alternatives. As the purpose here is not to compare strategies that differ in the absolute amount of information processed, the estimation will assume that $E F_{i}=E F_{i \text {, ideal }}$ for both strategies. Therefore, all relevant nonprice attributes and price are processed such that $m_{p i}=\left(m_{i}+1\right)$. Although this may be an unreasonable assumption, it provides a starting point from which to generate predictions and hypotheses.

The consumer's ability to recall attribute values at $i, \theta_{i}$, is operationalized at three levels, $0,0.5$, and 1 . This does not assume that $\theta_{i}$ increases linearly with knowledge or experience (M. Johnson 1985). Consumers may, for example, be able to recall abstract attribute values at relatively low levels of knowledge. Thus $\theta_{i}$ may be close or equal to one over a wide range of consumer knowledge for $i>0$.

Finally, values for the disutility parameters $\left(b_{1}\right.$ through $\left.b_{5}\right)$, which reflect the weight of the different effort components toward overall disutility, are required. There are two general procedures possible. The first is to substitute empirical estimates of these parameters obtained from previous studies. Unfortunately, such estimates are not available for all of the disutility parameters specified here. Existing empirical esimates may, in addition, be valid only in particular contexts (Chase 1978). An alternative procedure is to make reasonable assumptions about the values of these parameters. A common assumption is to equally weight the disutility parameters. This assumption is consistent with Newell and Simon's notion that total EIP's measure effort. It is also consistent with the "robust beauty" of unit weighted models in decision making (Dawes 1979).

While unit weighting may be reasonable for many parameters, one particular parameter deserves special attention. The effort to combine values across two qualitatively different attributes, $b_{5}$, may be much larger than the other effort disutilities. The disproportionate effort associated with across-attribute combinations is central to many theoretical discussions of strategy selection. After all, this is where consumers actually combine "apples and oranges" as opposed to, say, simply comparing apples. The relative ease of withinattribute processing purportedly rests on the ease of within-attribute comparisons relative to across-attribute combinations (Tversky 1969) and not on the fact that fewer EIP's are 
involved. Within-attribute comparisons only require a judgment of difference on an existing attribute while across-attribute combinations require the formation of a new attribute or dimension. This same reasoning may, of course, explain why consumers retain a within-attribute strategy when alternatives are noncomparable. Consumers may be willing to form a more abstract representation and perform a within-attribute strategy in order to minimize the number of across-attribute combinations necessary to evaluate noncomparable alternatives (M. Johnson 1984).

Several factors may affect the effort associated with this operation. For example, the more qualitatively different or dissimilar the attributes are the more effortful a combination of their values may be. When evaluating an automobile, it would seem easier to combine values across attributes like leg room and seat comfort than across leg room and gas mileage or seat comfort and gas mileage. Individual differences in analytical aptitude, such as those described by Christensen-Szalanski (1980), may also directly affect the size of this parameter. Combination effort may be relatively low for those individuals who are more skilled or simply have had more experience making combinations. On the other hand, individual and task related differences should have little effect on the effort to, for example, recall or compare values on attributes. As the effort to combine values across attributes may be central to strategy selection, equation (7) is estimated at increasing levels of $b_{5}$. As $b_{5}$ increases relative to other effort parameters, consistent with the theoretical arguments of both Tversky and $M$. Johnson, within-attribute processing should become more attractive.

Therefore, consistent with previous studies (Newell and Simon 1972; M. Johnson 1980; E. Johnson and Payne 1985), the effort disutility parameters $b_{1}$ through $b_{4}$ are simply unit weighted and set equal to one. Combination effort is, however, operationalized at four levels, $b_{5}=1,2,3$, and 4 . The values of all the parameters are presented in Table 1.

\section{Model Predictions}

Figures 1, 2, and 3 illustrate the models' predictions. In each figure, the difference in disutility between strategies, obtained by substituting operationalized parameter values into equation (7), is plotted against the level of abstraction at which the within-attribute strategy is performed from $i=0$ to $i=4$. This level increases with the noncomparability of the alternatives. The across-attribute strategy is always performed at $i=0$. Recall that disutility differences greater than zero imply use of the across-attribute strategy while differences less than zero imply use of the within-attribute strategy. When interpreting the figures, remember that the within-attribute strategy may be used at any level where comparability exists. Therefore, even though disutility may favor the across-attribute strategy at one level, the within-attribute strategy is predicted as long as the difference in disutility favors it at a higher level. When alternatives are comparable at level 0 , for example, as long as $E D\left(A_{0}\right)>E D\left(W_{i}\right)$ for some level $i$, consumers use the within-attribute strategy at that level. As long as, in addition, $E D\left(W_{i}\right)$ continues to decrease with increases in $i$, error/effort minimization predicts continued abstraction and use of the withinattribute strategy.

Case 1 , where attributes decrease linearly with $i$, is illustrated in Figure 1 for $n=2$. As the potential for $b_{5}$ (the disutility of making an across-attribute combination) to be relatively large is of central importance, Figure 1 illustrates the models' predictions where $b_{5}=4$. While not shown in the figure, an increase in combination disutility naturally favors the within-attribute strategy across levels of $i$ (and, implicitly, levels of comparability). This is consistent with the theoretical arguments of both Tversky (1969) and M. Johnson (1984). Interestingly, when $b_{5}=1$ (not shown), which is the value of the other effort parameters, there is no disutility difference between strategies at $i=0, n=2$ (i.e. 


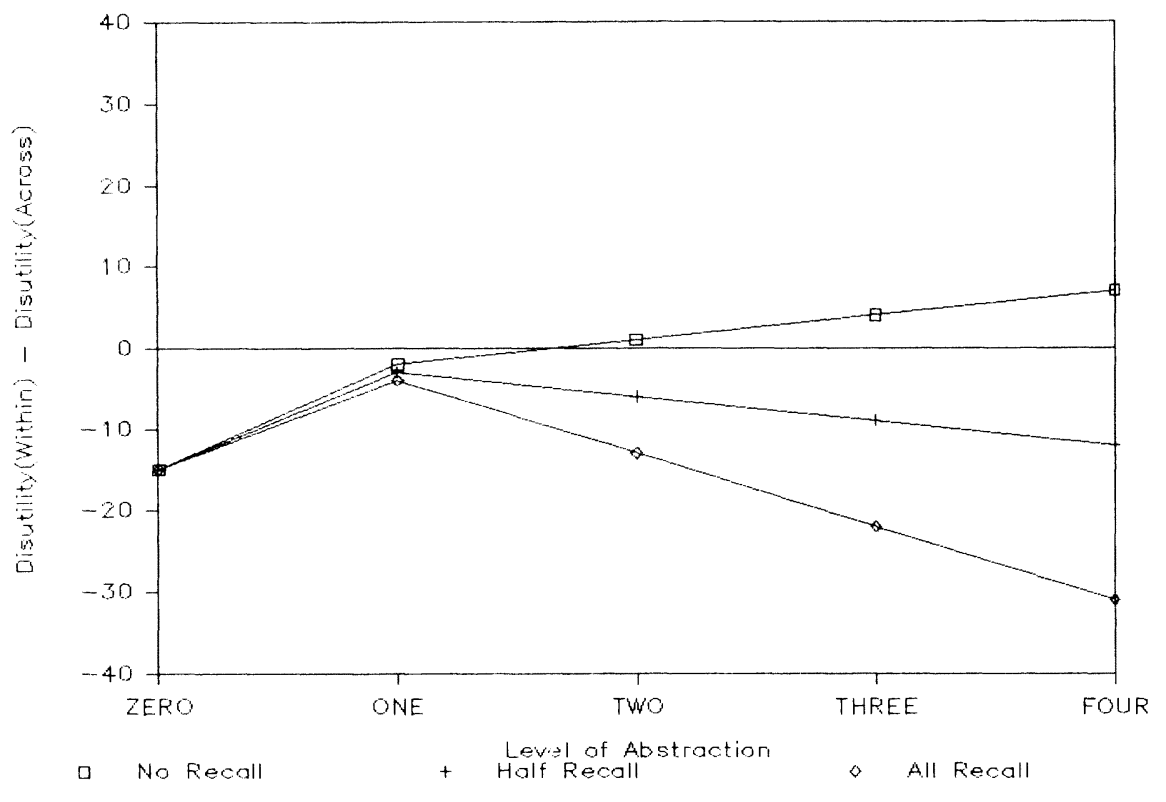

FIGURE 1. Case 1 (Linear Decrease): $b_{5}=4$.

equation $(7)=0$ ). Recall that preference for a within-attribute strategy when alternatives are comparable may be explained by assuming that combinations are more effortful than comparisons, not that fewer EIP's are involved. The prediction of the models is consistent with that explanation. Naturally, differences in combination effort across tasks and individuals should be explored further.

Figure 1 illustrates the affect of $\theta_{i}$, or the ability to recall attribute values, on the value of equation (7). Consider first alternatives that are comparable at $i=0$. When knowledge

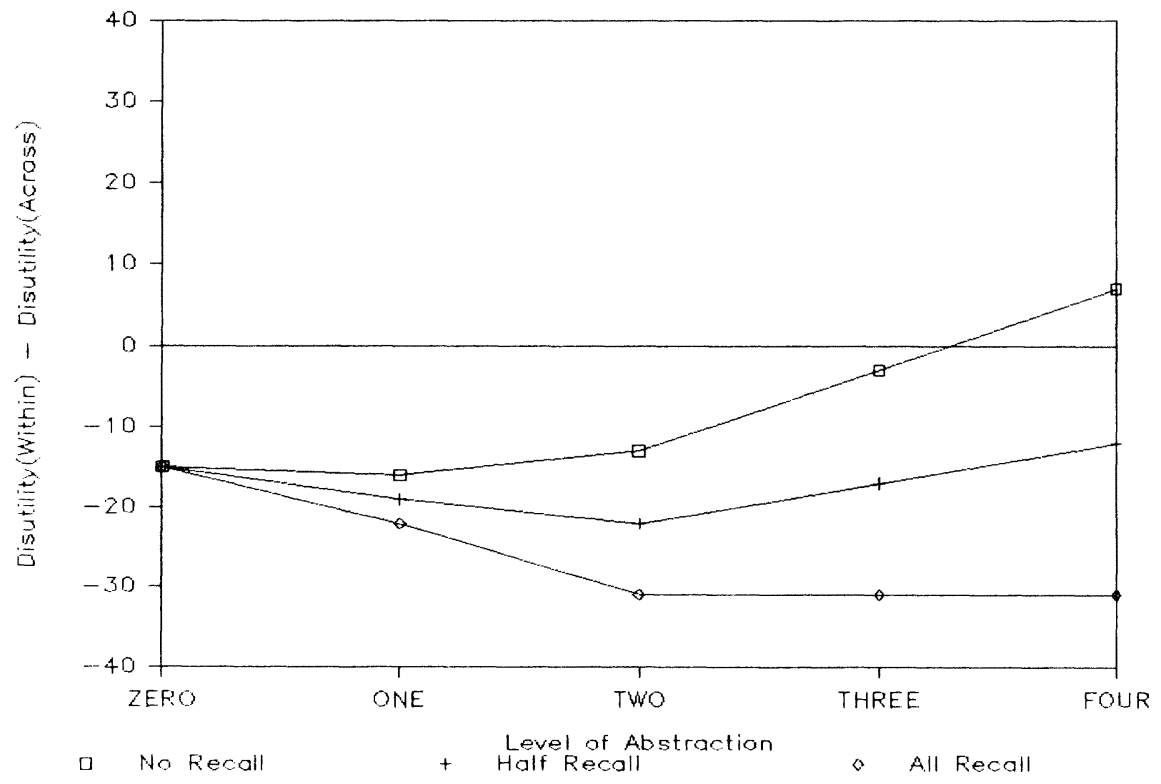

FIGURE 2. Case 2 (Drastic Decrease): $b_{5}=4$. 


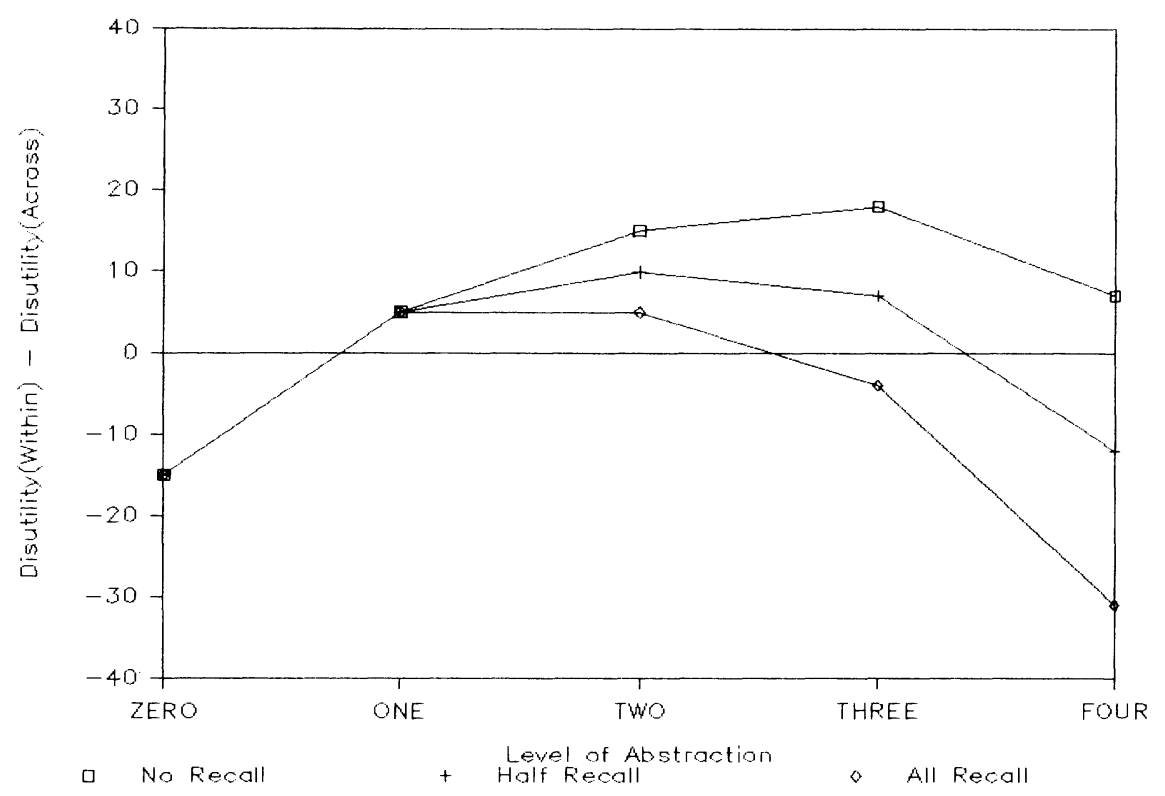

FIGURE 3. Case 3 (Gradual Decrease): $b_{5}=4$.

is low (no recall), consumers generally prefer to use the within-attribute strategy on concrete attributes. There is no incentive to form a more abstract representation. As $\theta_{i}$ increases, the difference in disutility between strategies decreases, favoring the withinattribute strategy. More importantly, the magnitude of this difference increases with $i$ such that higher levels of abstraction are generally preferred to lower levels even when alternatives are comparable. Therefore, when relative ability to recall is large, and resulting abstraction effort is small, the model predicts a general preference to abstract to $i_{\max }$ and use the within-attribute strategy, independent of comparability. This prediction is consistent with existing empirical results involving comparable alternatives, supporting the validity of the models. Consider that both the across-attribute strategy and the withinattribute strategy at higher levels of abstraction, while qualitatively different, are relatively "brand based" strategies. Bettman and Park (1980) show an increase in "brand" processing with consumer knowledge.

The comparability of alternatives and the number of relevant attributes from level to level have very predictable effects on strategy selection. Assuming combination disutility, $b_{5}$, is quite large, Figure 1 illustrates equation (7)'s predictions when relevant attributes decrease linearly from level to level and consumers differ in their ability to recall abstract attribute values. When recall is not possible, or $\theta_{i}=0$, consumers switch from using the within-attribute strategy at a level of abstraction where comparability exists to the acrossattribute strategy as the comparability of choice alternatives decreases (i.e. $i$ increases). When recall is completely possible, or $\theta_{i}=1$, the within-attribute strategy at $i=4$ is predicted for comparable and noncomparable alternatives alike.

Figures 2 and 3 present the results for the two remaining attribute cases, again assuming $b_{5}=4$ and $n=2$. Recall that Case 2 resembles nondurable products where $m_{i}=(5,2$, $1,1,1)$. The results for this case (see Figure 2) are similar to those for Case 1. When recall is not possible, consumers again switch from the within-attribute strategy at a level where comparability exists to the across-attribute strategy as alternatives become noncomparable. The switchover occurs, however, at a much higher level of noncomparability. Notice also that when attribute values are completely recallable, consumers are indifferent to the within-attribute strategy at $i=2,3$, or 4 for alternatives comparable at $i=0,1$, 
and 2. This observation, compared with the results of Case 1, reveals that decreases in effort for the within-attribute strategy from lower levels to higher levels depend on a corresponding decrease in relevant attributes.

Finally Case 3 , which resembles durable products where $m_{i}=(5,5,5,4,1)$, is presented in Figure 3. In this case, when recall of values is not possible, there is a very early shift from the within-attribute strategy to the across-attribute strategy as comparability decreases. When values are recallable, the within-attribute strategy at $i=4$ is generally preferred independent of comparability. Comparing Cases 2 and 3, the results suggest that the across-attribute strategy is more likely used when the choice involves durable products rather than nondurable products. This, of course, assumes that durables have more choice relevant, abstract attributes than nondurables. This result is due to the fact that there is more effort required to both build and evaluate abstract representations of consumer durables, where more abstract attributes are involved, than for nondurables at the same level of abstraction.

Overall, the models predict that as the comparability of choice alternatives decreases, consumers initially retain a within-attribute strategy and eventually switch to an acrossattribute strategy when ability to recall attribute values is low. This result is consistent with the empirical results reported in the M. Johnson (1984) study. Theoretically, the ease of within-attribute comparisons relative to across-attribute combinations gives consumers an incentive to retain a within-attribute strategy as comparability decreases. Eventually, the effort required to build more and more abstract representations causes a switchover to more across-attribute processing. When ability to recall attribute values is high, however, the within-attribute strategy is predicted at a high level of abstraction independent of comparability.

While not shown in the figures, the number of alternatives also has a very predictable effect on strategy selection. As $n$ increases, the difference in disutility between strategies increases. Equation (7) predicts that, in general, consumers prefer the withinattribute strategy when $n=2$ and $n=4$ but prefer the across-attribute strategy when $n=6$. While equation (7) does not apply to noncompensatory strategies, the prediction that the form of processing changes as the number of alternatives increases is consistent with the empirical results of the Lussier and Olshavsky (1979) study. In that study, consumers generally used an across-attribute, noncompensatory strategy, resembling the conjunctive rule, to reduce a large number of alternatives down to two or three. A compensatory within-attribute strategy, resembling additive difference, was typically used on the remaining alternatives or when only a small number of alternatives was involved.

Though also not presented here, these results are relatively insensitive to the levels of $a_{i}$ operationalized. Assuming three or ten levels rather than five does not greatly affect the predictions. This parameter only affects noncomparable alternatives, where abstraction is required, and only when abstract attribute values can not be recalled. The effect is to shift prediction toward the within-attribute strategy for more noncomparable alternatives as $a_{i \max }$ increases.

\section{Summary and Conclusions}

This study shows how theoretically based models of the difference between choice strategies allows predictions of consumer strategy selection for choices involving increasingly noncomparable alternatives. Naturally, the models and the results should be interpreted with caution. Many theoretical assumptions are made which may not hold in many choice situations. In particular, the error-effort trade-off may not describe strategy selection in many cases. Possible nonlinearities in the parameters and interactions among the parameters have also not been considered. In addition, only two strategies are modeled, and both assume compensatory information processing. 
Limitations aside, the models illustrate the important differences between the abstraction processes consumers use to compare more noncomparable alternatives. Three important, and testable, predictions regarding abstraction are also provided by operationalizing the models. First, the models predict that the use of the within-attribute strategy on noncomparable alternatives should increase as the effort to combine values acrossattributes, $b_{5}$, increases. It was suggested that combination effort is likely affected by both the dissimilarity of the attribute values being combined and individual differences across consumers. Future research should focus on measuring this operation and its effects on strategy selection. Second, operationalizing the strategy differences across different attribute cases suggests that the use of the across-attribute strategy on noncomparable alternatives increases as the number of relevant abstract attributes increases. When more relevant abstract attributes are involved, more effort is required to build an abstract representation and to evaluate alternatives using a within-attribute strategy. As a result, across-attribute processing on concrete-attributes may be more attractive. Here again the empirical effects of differences in the number of relevant attributes involved in choice should be directly addressed in future research. Third, situations exist in which consumers will form abstract representations and make abstract, within-attribute comparisons of alternatives even when the alternatives are already comparable on more concrete attributes. Because the number of relevant attributes may decrease from the concrete to the abstract, if a more abstract representation is easily formed, it may be easier to evaluate comparable alternatives on few, more abstract attributes than on several, more concrete attributes.

It is also encouraging that the models' predictions are consistent with the results of several empirical studies of decision making. For example, consistent with the empirical results in the M. Johnson (1984) study, the theoretical models predict that consumers initially retain a within-attribute strategy and eventually shift to an across-attribute strategy as the comparability of alternatives decreases (at least when ability to recall attribute values is low). Also, consistent with Lussier and Olshavsky (1979), the models predict that consumers will switch from within-attribute to across-attribute strategies as the number of alternatives in the choice increases. (This is also consistent with the predictions of other theoretical error/effort frameworks (E. Johnson 1979).) A third prediction, consistent with the empirical results of Bettman and Park (1980), is that the use of brand based strategies increases with consumer knowledge.

Finally, the model is very amenable to the inclusion of additional error and effort components or changes in existing components. The effect of processing different amounts of information can be studied. Other factors, such as whether or not higher level comparisons are more likely to result in decision error (M. Johnson 1984), could also be included in the models. If higher level comparisons are, in fact, less precise and lead to more error, consumers may not have the incentive to abstract to a maximum level of abstraction to use the within-attribute strategy when attribute values are easily recalled; consumers may make as concrete comparisons as possible. Obviously, further elaboration of the error side of the model should prove very useful. Our understanding of the consumer's strategy selection process benefits from a solid theoretical foundation. It is hoped that the analysis presented here is a step in that direction. ${ }^{4}$

Acknowledgement. The author thanks J. Edward Russo, Hillel J. Einhorn, Claes Fornell, Robert Libby, and two anonymous reviewers for their useful comments and Jerome Katrichis and Jill Johnson for assistance in programming and analysis. Financial assistance was provided by the University of Michigan's Graduate School of Business Administration.

\footnotetext{
${ }^{4}$ This paper was received August 1984 and has been with the author for 3 revisions.
} 


\section{References}

Beach, Lee Roy and Terence R. Mitchell (1978), “A Contingency Model for the Selection of Decision Strategies," Academy of Management Review, 3 (July), 439-449.

Bettman, James R. and C. Whan Park (1980), "Effects of Prior Knowledge and Experience and Phase of the Choice Process on Consumer Decision Processes," Journal of Consumer Research, 7 (December), 234248.

Boote, Arther S. (1975), “An Exploratory Investigation of the Roles of Needs and Personal Values in the Theory of Buyer Behavior," unpublished doctoral dissertation, Columbia University.

Brucks, Merrie (1985), "The Effects of Product Class Knowledge on Information Search Behavior," Journal of Consumer Research, 12 (June), 1-16.

Chase, W. (1978), "Elementary Information Processes," in W. K. Estes (ed.), Handbook of Learning and Cognitive Processes. Vol. 5. Hillsdale, N.J.: Erlbaum, 19-90.

Christensen-Szalanski, Jay J. J. (1978), "Problem Solving Strategies: A Selection Mechanism, Some Implications and Some Data," Organizational Behavior and Human Performance, 22, 307-323. (1980), "A Further Examination of the Selection of Problem-Solving Strategies: The Effects of Deadlines and Analytic Aptitudes," Organizational Behavior and Human Performance, 25, 107-122.

Collins, A. M. and E. F. Loftus (1975), “A Spreading-Activation Theory of Semantic Processing," Psychological Review, 82, 407-428.

Coombs, Clyde H. (1964), A Theory of Data, New York: Wiley.

Dawes, Robyn M. (1979), "The Robust Beauty of Improper Linear Models in Decision Making," American Psychologist, 34, 571-582.

Day, George S., Allan D. Shocker and Rajendra K. Srivastava (1979), "Customer-Oriented Approaches to Identifying Product-Markets," Journal of Marketing, 43 (Fall), 8-19.

Einhorn, Hillel J. (1979). "The Use of Nonlinear, Noncompensatory Models in Decision Making," Psychological Review, 73 (3), 221-230.

and Robin M. Hogarth (1975), "Behavioral Decision Theory: Processes of Judgment and Choice," Annual Review of Psychology, 32, 53-88.

Hogarth, Robin M. (1975), "Decision Time as a Function of Task Complexity," in Wendt/Vlek (eds.), Utility, Probability, and Human Decision Making. Dordrecht: Reidel.

Howard, John (1977), Consumer Behavior: Application of Theory. New York: McGraw-Hill.

Huber, Oswald (1980), "The Influence of Some Task Variables on Cognitive Operations in an InformationProcessing Decision Model," Acta Psychologica, 45, 187-196.

Johnson, Eric J. (1979), "Deciding How to Decide," paper presented at the Seventh Research Conference on Subjective Probability, Utility and Decision Making, August 27-31, Guteborg, Sweden. - and John Payne (1985), "Effort and Accuracy in Choice," Management Science, 395-414.

Johnson, Michael D. (1980), "An Information Processing Analysis of Product Labels," in Jerry Olson (ed.), Advances in Consumer Research, 7, 724-728.

- (1984), "Consumer Choice Strategies for Comparing Noncomparable Alternatives," Journal of Consumer Research, 11 (December), 741-753.

- (1985), "Inference by Abstraction," in Richard L. Lutz and Michael J. Houston (eds.), Winter Marketing Educators' Proceedings, Chicago: American Marketing Association; forthcoming.

Klein, Noreen M. (1983), "Utility and Decision Strategies: A Second Look at the Rational Decision Maker," Organizational Behavior and Human Performance, 31 (February), 1-25.

Lopes, Lola L. and Michael D. Johnson (1982), “Judging Similarity Among Strings Described by Hierarchical Trees," Acta Psychologica, 48, 13-26.

Lussier, Denis and Richard Olshavsky (1979), "Task Complexity and Contingent Processing in Brand Choice," Journal of Consumer Research, 6 (September), 154-165.

March, James G. (1978), "Bounded Rationality, Ambiguity, and the Engineering of Choice," Bell Journal of Economics, 9 (2), 587-608.

McAllister, D. W., Terence Mitchell and Lee Roy Beach (1979), "The Contingency Model for the Selection of Decision Strategies: An Empirical Test," Organizational Behavior and Human Performance, 24, 228-244.

Newell, Alan and Herbert Simon (1972), Human Problem Solving. Englewood Cliffs, N.J.: Prentice-Hall.

Payne, John (1976), "Task Complexity and Contingent Processing in Decision Making: An Information Search and Protocol Analysis," Organizational Behavior and Human Performance, 16 (August), 366-387.

Quillian, M. R. (1969), “The Teachable Language Comprehender: A Simulated Program and Theory of Language," Communications of ACM, 12, 459-476.

Rokeach, Milton J. (1973), The Nature of Human Values. New York: Wiley.

Russo, J. Edward (1981), "The Decision to Use Product Information at the Point of Purchase," in Ronald W. Stampfl and Elizabeth C. Hirshman (eds.), Theory in Retailing: Traditional and Nontraditional Sources. Chicago: American Marketing Association, 155-167. 
and Barbara A. Dosher (1983), "Strategies for Multialternative Binary Choice," Journal of Experimental Psychology: Learning, Memory and Cognition, 9 (4), 676-696.

- and Larry Rosen (1975), “An Eye Fixation Analysis of Multi-Alternative Choice," Memory and Cognition, 3 (3), 267-276.

Sheluga, David, James Jaccord and Jacob Jacoby (1979), "Preference, Search, and Choice: An Integrative Approach,” Journal of Consumer Research, 6 (September), 166-176.

Shugan, Steven (1980), “The Cost of Thinking," Journal of Consumer Research, 7 (September), 99-111.

Slovic, Paul and Douglas MacPhillamy (1974), "Dimensional Commensurability and Cue Utilization in Comparative Judgment," Organizational Behavior and Human Performance, 11, 172-194.

Smith, James F., Terence R. Mitchell and Lee Roy Beach (1982), "A Cost-Benefit Mechanism for Selecting Problem-Solving Strategies: Some Extensions and Empirical Tests," Organizational Behavior and Human Performance, 29, 370-396.

Srivastava, Rajendra K., Mark I. Alpert and Allen D. Shocker (1984), “A Customer-Oriented Approach for Determining Market Structures,” Journal of Marketing, 48 (Spring), 32-45.

Thorngate, Warren (1980), "Efficient Decision Heuristics," Behavioral Science, 25, 219-225.

Tversky, Amos (1969), "Intransitivity of Preferences," Psychological Review, 76 (1), 31-48. (1972), "Elimination by Aspects: A Theory of Choice," Psychological Review, 79 (4), 281-299.

and Daniel Kahneman (1974), “Judgment Under Uncertainty: Heuristics and Biases,” Science, 185, 1124-1131.

Wright, Peter L. (1975), “Consumer Choice Strategies: Simplifying vs. Optimizing,” Journal of Marketing Research, 12 (February), 60-67.

Yates, J. Frank, Carolyn Jagacinski and Mark D. Faber (1978), "Evaluation of Partially Described Multiattribute Options," Organizational Behavior and Human Performance, 21, 240-251. 